Article

\title{
Dynamics of Jasmonate Metabolism upon Flowering and across Leaf Stress Responses in Arabidopsis thaliana
}

\author{
Emilie Widemann ${ }^{1,+}$, Ekaterina Smirnova ${ }^{1,+}$, Yann Aubert ${ }^{1}$, Laurence Miesch ${ }^{2}$ and \\ Thierry Heitz ${ }^{1, *}$ \\ Received: 7 December 2015; Accepted: 28 December 2015; Published: 6 January 2016 \\ Academic Editor: Debora Gasperini \\ 1 Institut de Biologie Moléculaire des Plantes, CNRS-UPR2357, associée à l'Université de Strasbourg, \\ 12 rue du Général Zimmer, 67084 Strasbourg Cedex, France; widemann@mail.tsinghua.edu.cn (E.W.); \\ ekaterina.smirnova@ibmp-cnrs.unistra.fr (E.S.); ian.aubert@hotmail.fr (Y.A.) \\ 2 Laboratoire de Chimie Organique Synthétique, Unité Mixte de Recherche 7177, Université de Strasbourg, \\ 67008 Strasbourg Cedex, France; lmiesch@unistra.fr \\ * Correspondence: thierry.heitz@ibmp-cnrs.unistra.fr; Tel.: +33-367155358; Fax: +33-367155300 \\ + These authors contributed equally to the paper.
}

\begin{abstract}
The jasmonic acid (JA) signaling pathway plays important roles in adaptation of plants to environmental cues and in specific steps of their development, particularly in reproduction. Recent advances in metabolic studies have highlighted intricate mechanisms that govern enzymatic conversions within the jasmonate family. Here we analyzed jasmonate profile changes upon Arabidopsis thaliana flower development and investigated the contribution of catabolic pathways that were known to turnover the active hormonal compound jasmonoyl-isoleucine (JA-Ile) upon leaf stress. We report a rapid decline of JA-Ile upon flower opening, concomitant with the massive accumulation of its most oxidized catabolite, 12COOH-JA-Ile. Detailed genetic analysis identified CYP94C1 as the major player in this process. CYP94C1 is one out of three characterized cytochrome P450 enzymes that define an oxidative JA-Ile turnover pathway, besides a second, hydrolytic pathway represented by the amido-hydrolases IAR3 and ILL6. Expression studies combined with reporter gene analysis revealed the dominant expression of CYP94C1 in mature anthers, consistent with the established role of JA signaling in male fertility. Significant CYP94B1 expression was also evidenced in stamen filaments, but surprisingly, CYP94B1 deficiency was not associated with significant changes in JA profiles. Finally, we compared global flower JA profiles with those previously reported in leaves reacting to mechanical wounding or submitted to infection by the necrotrophic fungus Botrytis cinerea. These comparisons revealed distinct dynamics of JA accumulation and conversions in these three biological systems. Leaf injury boosts a strong and transient JA and JA-Ile accumulation that evolves rapidly into a profile dominated by $\omega$-oxidized and/or Ile-conjugated derivatives. In contrast, $B$. cinerea-infected leaves contain mostly unconjugated jasmonates, about half of this content being $\omega$-oxidized. Finally, developing flowers present an intermediate situation where young flower buds show detectable jasmonate oxidation (probably originating from stamen metabolism) which becomes exacerbated upon flower opening. Our data illustrate that in spite conserved enzymatic routes, the jasmonate metabolic grid shows considerable flexibility and dynamically equilibrates into specific blends in different physiological situations.
\end{abstract}

Keywords: Arabidopsis; JA-Ile; cytochrome P450; CYP94; amidohydrolase; hormone homeostasis; jasmonate catabolism 


\section{Introduction}

Jasmonic acid (JA) and its derivatives, commonly referred to as jasmonates (JAs), are lipid-derived phytohormones with a plethora of functions. JAs play important roles in plant growth and development as well as in plant biotic and abiotic stress responses in cooperation with other plant hormones [1-4].

The details of JA biosynthesis in A. thaliana have been extensively described (e.g., in [5]). Briefly, JA synthesis starts in plastid membranes when phospholipases $A_{1}$ like Deficient in Anther Dehiscence 1 (DAD1) or related enzymes releases $\alpha$-linolenic acid (C18:3) [6,7], which then undergoes oxidation by a 13-lipoxygenase into 13-hydroperoxy-octadecatrienoic acid (13-HPOT). By the successive action of 13-allene oxide synthase (AOS) and allene oxide cyclase (AOC), 13-HPOT is converted to 12-oxo-phytodienoic acid (OPDA). OPDA is then transferred to peroxisomes, where its cyclopentenone ring is reduced by OPDA reductase 3 (OPR3) and its carboxylic side chain undergoes three rounds of beta-oxidation involving acyl-CoA oxidase (ACX1) to yield JA.

Upon stress or developmental signals, JA can be channeled through at least 6 different metabolic routes $[4,8]$ thus generating a vast array of compounds, some of them being associated with distinct biological activities [9]. How jamonate fluxes and different compound pool sizes are controlled remains largely unknown. Part of the accumulated JA is conjugated to various amino acids via the action of JAR1 or related enzymes, and one major conjugate, jasmonoyl-isoleucine (JA-Ile), represents a bioactive form of the hormone $[10,11]$. JA-Ile specifically promotes the assembly of a co-receptor composed of COI1 (CORONATINE INSENSITIVE1), an F-box protein component of the E3 ubiquitin ligase SCFCOI, and transcriptional repressors of the JAZ (JASMONATE ZIM-DOMAIN) protein family. In the absence of JA-Ile, JAZs along with a number of associated co-repressors, bind to and block transcription factors (e.g., MYC2/3/4) [12] that target downstream genes involved in specific JA responses. The assembly of the COI1-(JA-Ile)-JAZ complex triggers the ubiquitination of JAZs proteins leading to their proteolytic degradation via the ubiquitin/26S proteasome pathway [11], ultimately releasing the transcription of JA-responsive genes from repression.

Different JAs derivatives have long been known to accumulate differentially in various plant organs or physiological situations. The formation of some of these derivatives was initially proposed to represent JA inactivation pathways [13]. Later, the identification of JA-Ile as the bioactive signal mediating most COI1-mediated JA responses has renewed interest for metabolic studies, particularly those addressing JA-Ile turnover mechanisms. As JA-Ile is a master switch orchestrating broad genetic reprogramming, and antagonizing other hormonal responses [14], its level need to be tightly controlled to provide flexible and reversible stress or developmental signaling. Two enzymatic pathways for JA-Ile removal have recently been identified and operate by enzymatic oxidation of the JA moiety (JA-Ile oxidative pathway) or by cleavage of the amide bond between JA and Ile (JA-Ile hydrolytic/deconjugation pathway), repectively. On the one hand, oxidation of JA side chain terminal carbon (hence the term w-oxidation) is achieved by the action of cytochromes P450 of the CYP94 family, with CYP94B3 and CYP94C1 catalyzing preferential formation of 12OH-JA-Ile and 12COOH-JA-Ile, respectively. We and others have extensively analyzed the genetics and biochemistry of this oxidative pathway (Figure 1). CYP94 induction in part controls the duration and amplitude of the JA-Ile pulse upon leaf stress and consequently attenuates defense and resistance responses to herbivore and microbial attacks [15-18]. CYP94-mediated $\omega$-oxidation of JA-Ile results in a strong decrease of its hormonal activity. Indeed, whereas $12 \mathrm{OH}-\mathrm{JA}$-Ile is still able to weakly promote the formation of COI1-JAZ complexes or to induce detectable transcriptional responses, 12COOH-JA-Ile is completely inactive $[15,19]$. CYP94 catalysis may be even more diversified as CYP94B1, B3 and C1 enzymes can also oxidize other JA conjugates including JA-valine or JA-phenylalanine [20,21], and CYP94C1 activity generates 12CHO-JA-Ile aldehyde in planta [21]. 


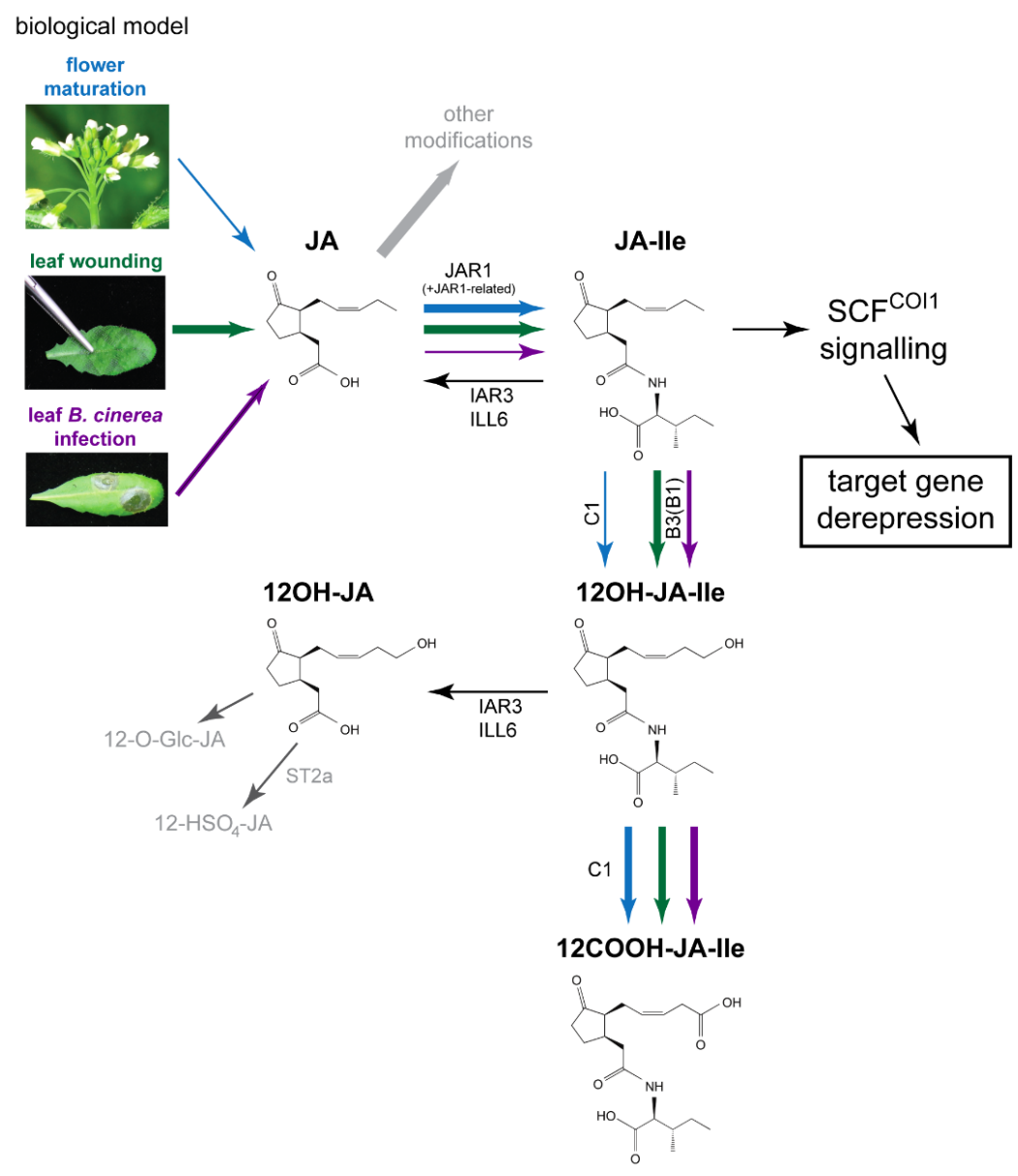

Figure 1. Proposed model for interconversion routes between jasmonic acid (JA) and its Ile-conjugated / $\omega$-oxidized derivatives upon leaf stress responses or flower maturation, with emphasis on quantitative aspects as explained in main text. Upon leaf stress responses (wounding or B. cinerea infection) or flower maturation JA synthesis is activated to different extents, mechanical wounding providing the strongest burst. JA can be modified via different metabolic routes, one being the formation of jasmonoyl-isoleucine (JA-Ile) by JAR1 or related conjugating enzymes. JA-Ile is then either $\omega$-oxidized by the action of CYP94 family enzymes to its hydroxy- (12OH-JA-Ile) or carboxy- (12COOH-JA-Ile) derivatives. In addition, JA-Ile and $12 \mathrm{OH}-\mathrm{JA}-\mathrm{Ile}$ can be hydrolyzed by the amidohydrolases IAR3 and ILL6, leading to the formation of JA and 12OH-JA, respectively. The thickness of arrows for the main JA metabolic pathway (from JA to 12COOH-JA-Ile) reflects the abundance of a given reaction product in each physiological context as detailed in Discussion. Comparative analysis of jasmonate profiles in the three biological systems as described in this paper shows that adequate JA-Ile levels can be achieved with distinct upstream and downstream metabolite pool sizes through the action of common enzymes. Blue-flower maturation, green-leaf wounding, purple-leaf B. cinerea infection. Predominant enzymes performing a given reaction are mentioned as follows: CYP94C1: C1, CYP94B1: B1, CYP94B3: B3, ST2a-sulfotransferase 2a. The compounds are shown in $(3,7)$-cis stereochemistry.

On the other hand, the JA-Ile deconjugation or hydrolytic pathway was first identified in the wild tobacco Nicotiana attenuata where JASMONOYL-ISOLEUCINE HYDROLASE 1 (JIH1) encodes an amidohydrolase (AH) whose silencing strongly enhanced JA-Ile levels and several JA-Ile-controlled direct and indirect defenses [22]. In Arabidopsis, IAR3 and ILL6, two JIH1 orthologous genes exhibit a strong co-regulation with the JA pathway. IAR3 was initially described as cleaving exogenously-fed auxin-amino acid conjugates, liberating active auxin in planta during seed germination [23]. However, in stressed leaves, JA conjugates are much more abundant than auxin conjugates, and genetic 
analysis indicated that induced IAR3 and ILL6 activity acts preferentially on JA-Ile and 12OH-JA-Ile substrates [24]. The identification of this latter reaction provided the first, indirect biosynthetic route towards tuberonic acid (12OH-JA) formation (Figure 1) and its sulfated and glucosylated derivatives. The oxidative and hydrolytic JA-Ile catabolic pathways thus work coordinately to attenuate JAs signaling [19] and as a consequence shape the dynamic JAs signatures in defending leaves $[9,15,16]$.

Plant reproduction has been recognized as a developmental process where the JA pathway is crucial. Long-standing genetic evidence in Arabidopsis has shown that most mutants in JA synthesis (fad378, dad1, aos, opr3 acx1/5) and perception (coi1) are male sterile [7,25-30], due to defects in late stage of Arabidopsis flower development. These genotypes are impaired in filament elongation, pollen maturation and finally anther dehiscence, thereby preventing well-timed pollination of the pistil $[7,31,32]$. Cumulative evidence has elucidated complex hormonal networks which orchestrate flower development and maturation [33,34]. Auxin-regulated events promote JA biosynthesis in stamens that in turn activates a complex of bHLH-MYB transcription factors (TFs) involving MYC2/3/4/5 [35] and MYB21/24. These latter are two JA-inducible TFs that are guarded by JAZ repressors [36] and that were initially identified as governing proper stamen development [37]. The general requirement of JA signaling in flower development and fertility was more recently found conserved in other plant species, but striking variations were evidenced. For example, in rice, defects along the metabolic or signaling pathway affect floret opening and anther dehiscence, but also spikelet development [38-40]. In maize, JA biosynthetic mutants show even earlier flower developmental defects including sex determination of male reproductive organs [41,42]. A different situation is known in tomato, where the jai1 mutant that is defective for the homolog of the F-box protein COI1 is female sterile [43]. More recent analysis seems to involve OPDA as the specific signal required for proper embryo development [44]. Finally, in the wild tobacco N. attenuata, JA-Ile coordinates metabolic networks required for anthesis and floral attractant emission [45].

Consistent with this genetic evidence for important roles in plant reproduction, many JAs have been described as accumulating abundantly in flowers from different species [46]. Early investigations in tomato revealed a specific jasmonate blend in distinct flower organs [47]. All these data pre-dated the identification of JA-Ile as a major signal in this hormonal class or the still uncomplete unraveling of JA-Ile catabolic pathway. However, in contrast to their analysis in leaf stress responses, dynamics of JA-Ile metabolism during the crucial milestone that represents flower development have not been investigated in any plant species so far.

Here we present an investigation of JA-Ile and JAs dynamics in Arabidopsis flowers. Results demonstrate that CYP94C1-mediated JA-Ile oxidation occurs at Arabidopsis flower opening. We then compare these data with JA dynamics previously determined in leaves reacting to mechanical wounding or to infection by the fungus Botrytis cinerea. Despite being the site of active JA-Ile metabolism and signaling, these three biological models develop clearly distinct JA profiles, reflecting different limiting steps and fluxes through conjugation/deconjugation and $\omega$-oxidation processes.

\section{Results}

\subsection{Oxidation of JA-Ile Increases at Flower Opening}

Arabidopsis flowers were collected as two separate pools : closed flower buds corresponding to stages 1-12 defined by [32], and opening flowers of stages 13-14 (Figure 2A). Metabolites were extracted and samples analyzed quantitatively by UPLC-MS/MS for their contents in JA, 12OH-JA, JA-Ile and the two oxidized derivatives 12OH-JA-Ile and 12COOH-JA-Ile (Figure 2B). All compounds were readily detected both in closed buds and open flowers, the most abundant compounds being JA in the former and $12 \mathrm{COOH}-\mathrm{JA}-I l e$ in the latter. 12OH-JA and 12OH-JA-Ile levels were stable upon flower opening. In contrast, drastically reduced JA-Ile quantities were recorded in open flowers, simultaneous with a large increase in $12 \mathrm{COOH}-\mathrm{JA}-\mathrm{Ile}$ abundance that reaches $2 \mathrm{nmol} \cdot \mathrm{g}$. $\mathrm{FW}^{-1}$. These temporal evolutions suggest that an active JA-Ile oxidation process to $12 \mathrm{COOH}-\mathrm{JA}-\mathrm{Ile}$ takes place 
at flower opening. The low and steady content in 12OH-JA-Ile may indicate that this intermediate substrate is rapidly turned over toward the more oxidized conjugate and/or cleaved to $12 \mathrm{OH}-\mathrm{JA}$ by amidohydrolases.

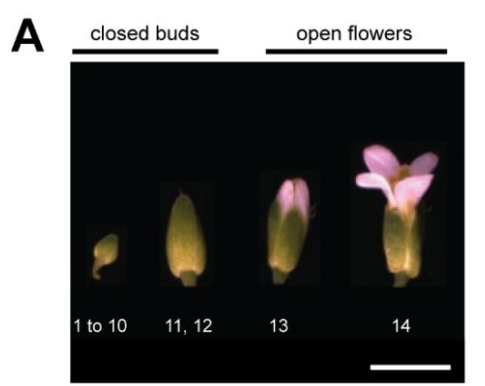

B
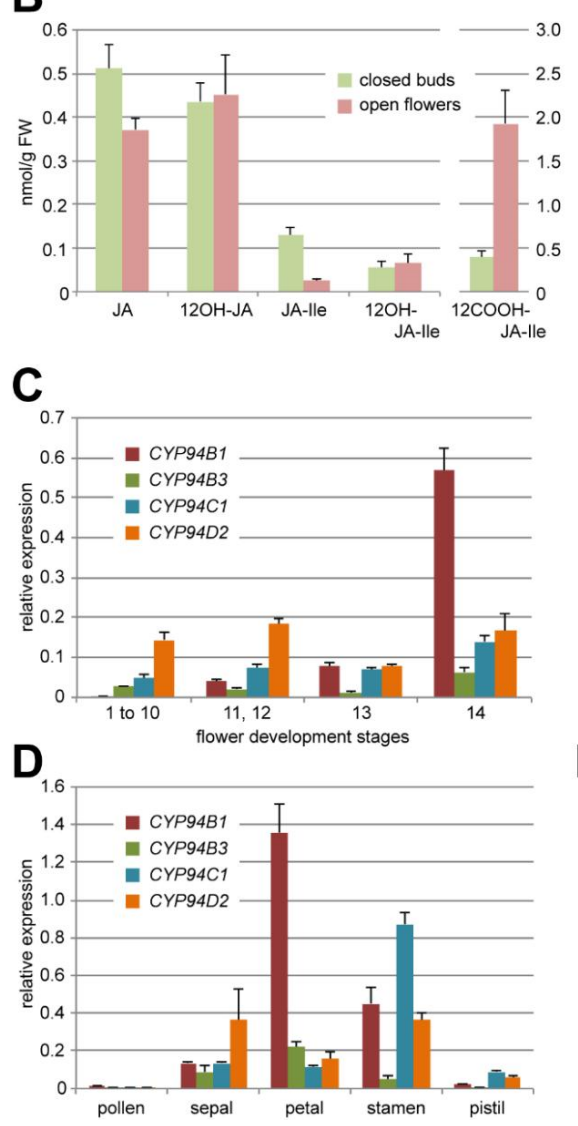

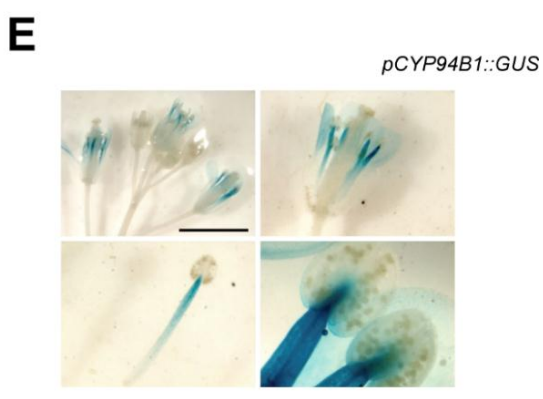

pCYP94C1::GUS
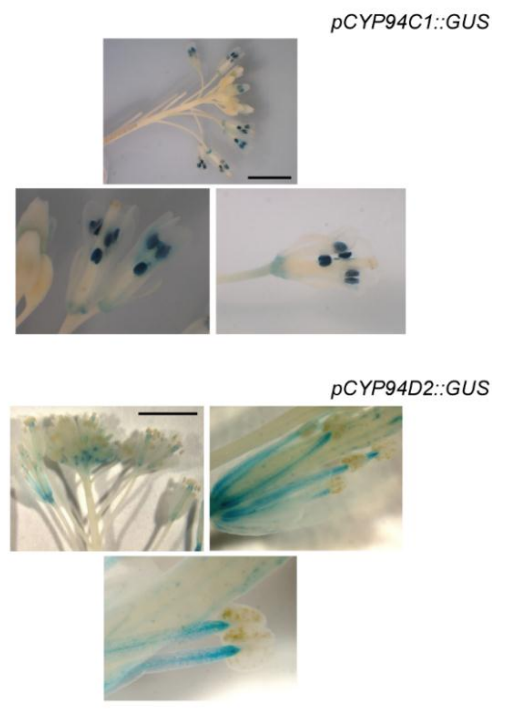

$\mathbf{F}$

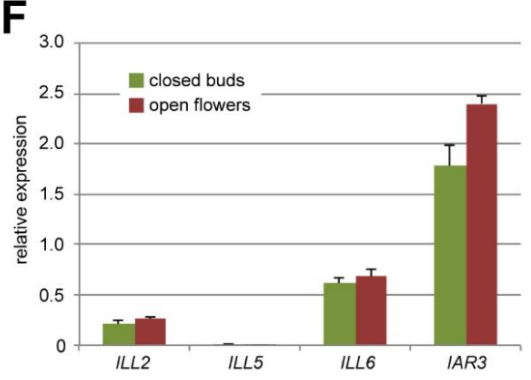

Figure 2. (A) A. thaliana flower development stages corresponding to that indicated in [32]. Stages 1-12 further referred to closed buds, stages 13 and 14-to open flowers (as indicated above). Scale bar corresponds to $3 \mathrm{~mm}$. (B) UPLC-MS/MS quantification of five JAs content in Col0 closed flower

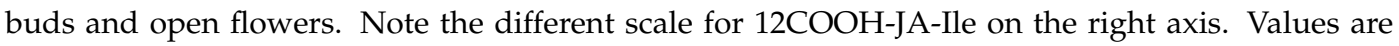
presented as means with SD of three independent biological repeats. (C, D) Relative expression levels of 4 CYP94 genes $(-B 1,-B 3,-C 1,-D 2)$ in Col0 flowers distributed by development stages (C) or flower organs (stade 14)(D). Expression levels are normalized by expression of two reference genes. Values are presented as means with SD of three technical repeats. (E) Typical GUS staining pattern of flowers of pCYP94C1::GUS, pCYP94B1::GUS and pCYP94D2::GUS transgenic lines at different magnitude (from whole inflorescence to a separate anther). Two independent lines were analyzed for each construct. Scale bar corresponds to $3 \mathrm{~mm}$. (F) Relative expression levels of 4 AH genes (ILL2, ILL5, ILL6, IAR3) in Col0 closed flower buds or open flowers. Expression levels are normalized by expression of two reference genes. Values are presented as means with SD of three technical repeats. 


\subsection{JA-Ile Catabolic Genes Display Dynamic Expression upon Flower Development}

\subsubsection{The JA-Ile Oxidase-Encoding Genes CYP94B1, CYP94B3 and CYP94C1 Are Differentially} Expressed at Flower Opening

Expression of the six genes in the CYP94 family was analyzed by real-time PCR in flowers collected at a higher temporal resolution, in 4 samples covering all stages of development from floral meristem up to open flowers (stage 1 to 10,11-12,13,14). CYP94D1 yielded no signal and CYP94B2 expression was very weak. Consequently, these genes were not further studied. The expression of the other 4 genes (CYP94B1, B3, C1 and D2) was reliably detected in all samples. CYP94D2 is a gene of unknown function which does not encode a JA-Ile oxidase; its global expression fluctuated within a two-fold range through the different stages of developing flowers. Interestingly, the expression of CYP94B1,-B3 and $-C 1$, all known as encoding JA-Ile oxidases acting in stressed leaves [15-19] peaked at stage 14 open flowers (Figure 2C), concomitant with JA-Ile oxidation (Figure 2B). CYP94B3 was the weakest expressed gene, whereas CYP94B1 expression was most prominent. When flower organs from stage 14 were dissected and analyzed, CYP94B1 expression was highest in petals and stamens (Figure 2D) whereas CYP94C1 expression appeared largely stamen-specific. All four genes were very weakly expressed in pollen. Finally, promoter-reporter transformants were generated for CYP94B1, C1 and $D 2$ and analyzed for $\beta$-glucuronidase (GUS) expression in inflorescences. Representative patterns are shown in Figure 2E. $p$ CYP94B1-GUS displayed a diffuse staining in distal part of petals and a gradually more dense signal in the upper part of elongating stamen filaments, confirming earlier observations by [19]. In contrast, $p C Y$ P94C1-GUS marked exclusively and strongly anthers in open flowers at stages 13-14. $p$ CYP94D2-GUS stained young flower bud stalks and later vascular tissues in elongated anther filaments of open flowers. These data reveal organ-specific expression patterns that are consistent with qPCR results and point to a possible role of some CYP94 genes in JA-Ile turnover in Arabidopsis flowers.

\subsubsection{Amidohydrolases Are Expressed at the Same Level in Closed Buds and Open Flowers}

As IAR3 and ILL6, two members of the AH enzyme family, act in JA-Ile turnover in concert with CYP94 enzymes upon leaf wounding $[19,24]$, we investigated their global expression profiles by RT-qPCR in closed and open ColO flowers (Figure 2F). Among the 7 members of the AH family we also analyzed ILL5 as a third specimen of JA-coregulated gene, and ILL2 for which no link with the JA pathway is known. For ILL5 no expression was found at either stage. For the other 3 analyzed genes, expression levels were largely unchanged upon floral development. ILL2 displayed low expression, while IAR3 exceeded ILL6 expression and was slightly increased at flower opening. These results are consistent with $A H$ gene expression reported previously in Arabidopsis inflorescences [23].

\subsection{CYP94C1 Is the Major Enzyme for JA-Ile Oxidation upon Arabidopsis Flower Opening}

As increased expression of some CYP94 genes at flower opening-particulary in stamens-correlated with active JA-Ile oxidation, we determined the impact of CYP94 deficiency on unoxidized or oxidized JA-Ile levels. Metabolic extracts of closed flower buds (stages 1-12) or opening flowers (stages 13-14) from single, double, and triple mutants in CYP94B1, $-B 3$ and -C1 genes and also p35S-CYP94C1 overexpressing plants (hereafter C1-OE) were submitted to JA-Ile profiling by UPLC-MS/MS analysis. Surprisingly, all loss-of-function mutants displayed near-WT JA-Ile levels at the two developmental stages analyzed. In contrast, the C1-OE line showed a significant $(70 \%)$ decrease of JA-Ile content in closed buds but barely reduced in open flowers compared to WT (Figure 3A). 12OH-JA-Ile, the initial JA-Ile oxidation product, was accumulated to low levels, which were further depressed to variable extents in the different mutants, notably in cyp $94 c 1$ and cyp94b1b3. This indicates that all three CYP94 genes act redundantly in the formation and build-up of this intermediate. Furthermore, its levels are more strongly reduced in $C 1-O E$ open flowers, indicating a higher consumption. Finally, the levels of $12 \mathrm{COOH}-\mathrm{JA}-\mathrm{Ile}$, the end product of CYP94-dependent JA-Ile oxidation pathway were reduced to various entents in open flowers of CYP94C1-deficient 
lines $(c 1, b 1 c 1, b 3 c 1$ and $b 1 b 3 c 1)$ (Figure 3C). Reciprocally, ectopic CYP94C1 overexpression enhanced 12COOH-JA-Ile abundance in closed buds as well as in open flowers compared to WT. These results indicate that, as in fungus-infected leaves [15], CYP94C1 is the major enzyme for oxidative JA-Ile catabolism upon flower maturation. Unexpectedly, when the cyp $94 c 1$ mutation was combined with cyp94b1 (in $b 1 c 1$ and $b 1 b 3 c 1$ ), 12COOH-JA-Ile levels were higher in open flowers than in lines where CYP94B1 was functional (in $c 1$ and $b 3 c 1$ ). This observation indicates the possibility of an alternative route that may substitute to CYP94 deficiency to make 12COOH-JA-Ile [48].

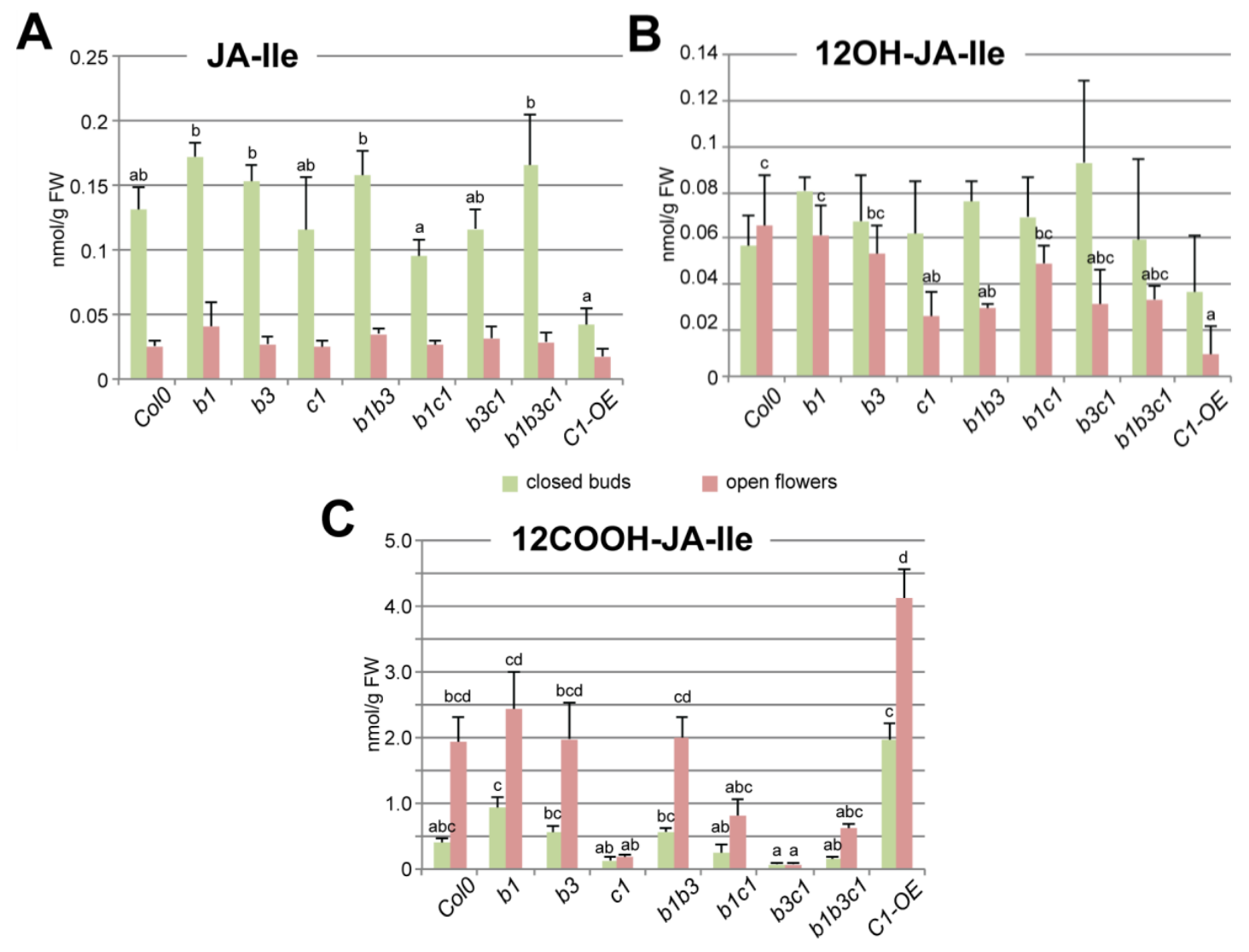

Figure 3. UPLC-MS/MS analysis of conjugated JAs (JA-Ile, 12OH-JA-Ile and 12COOH-JA-Ile) abundance in closed buds or open flowers of Col0 control plants and of simple mutants cyp94b1, $-b 3$ and $-c 1$, double mutants cyp94b1b3, -b1c1 and -b3c1, triple mutant cyp94b1b3c1, and CYP94C1 over-expressor mutant (C1-OE). Values are presented as means with SD of three independent biological repeats. Statistical analysis was applied separately to the pool of data corresponding to either closed buds or open flowers. Columns are labelled with different letters indicating a significant difference in given jasmonate content between genotypes as determined by Kruskal-Wallis one-way analysis of variance (ANOVA) $(p<0.05)$. Absence of letters corresponds to the absence of significant difference in a given JA abundance in different analyzed genotypes.

Of note, no obvious flower developmental defects were recorded for any of the CYP94-modified lines, in contrast to CYP94B1-OE and CYP94B3-OE lines reported previously as male sterile in $[18,19]$. Very high expression in these latter lines may deplete JA-Ile below a threshold level required for proper flower development. 


\section{Discussion}

\subsection{CYP94C1 Is a Major Player in JA-Ile Oxidation upon Floral Opening}

JA-Ile inactivation mechanisms have been recently characterized, mostly in leaf stress responses where CYP94 and amidohydrolase $(A H)$ genes are induced together with JA biosynthetic and signaling genes [15-19,24]. These catabolic pathways inactivate the hormone by $\omega$-oxidation or conjugate cleavage and thereby attenuate defense responses. They also largely remodel jasmonate metabolic profiles (or jasmonate signatures) in attacked tissues. The precise implications of the accumulation of various JAs deriving from JA-Ile breakdown remain to be determined. In wounded leaves rapid JA-Ile inactivation/removal is achieved by the concerted action of CYP94 and AH enzymes. Under this stimulus, CYP94B3 and CYP94C1 work sequentially: CYP94B3 contributes predominantly to the initial oxidation to 12OH-JA-Ile and CYP94C1 accounts for the conversion of this intermediate to 12COOH-JA-Ile [16]. AH not only deconjugates JA-Ile but also acts on 12OH-JA-Ile to generate $12 \mathrm{OH}-\mathrm{JA}$ [24]. In contrast, in leaves reacting to fungal infection by Botrytis cinerea, CYP94C1 was the predominant member controlling hormone oxidation [15]. The genetic and biochemical dissection of these two biological models has defined a complex jasmonate metabolic grid where JA-Ile acts as a metabolic hub directing the synthesis of numerous conjugated and unconjugated derivatives [9].

Here we used the same genetic and analytical tools to investigate JA-Ile turnover in Arabidopsis floral development. We show that at flower opening, a rapid decrease in JA-Ile levels matches the massive accumulation of $12 \mathrm{COOH}-\mathrm{JA}-I l e$. These results corroborate similar profiles obtained in the frame of the study of CYP715A1 with the methods we developed here [48]. The present genetic analysis of the CYP94 family revealed that CYP94C1 alone accounts for this conversion, with a comparatively low pool size of the intermediate 12OH-JA-Ile. Ectopic CYP94C1 overexpression had the most (relative to WT) impact in closed flower buds, when native CYP94C1 expression is still low. In contrast to the situation in wounded leaves, we did not detect a significant contribution of CYP94B3 to JA-Ile homeostasis in flowers. Remarkably, no significant increase of JA-Ile level was observed in any analyzed mutated line relative to WT even in the double cyp94b3c1 mutant where 12COOH-JA-Ile formation was virtually abolished. Additionally, the drop in JA-Ile levels in open flowers was similar to WT in all lines. This indicates that in contrast to injured leaves where the CYP94 pathway is essential for rapid clearance of JA-Ile [16], flowers are able to eliminate the hormone through other means. Compensation of CYP94-deficiency by a higher expression of the AH pathway would be a possibility, but IAR3 and ILL6 transcript levels were not found significantly perturbed in flowers of CYP94-deficient plants (data not shown).

The impact of the different mutations on global flower JA-Ile homeostasis is consistent with known enzymatic characteristics of the three CYP94 proteins [16] and with their expression profiles. The use of CYP94 promoter-reporter lines provided novel insights into possible sites of JA-Ile turnover. $p$ CYP94B1::GUS reporter labelled elongated filaments and petals, in accordance with JA being synthesized in young filaments $[7,49]$ to promote their elongation and anther dehiscence. CYP94B1 expression may therefore initiate signal deactivation during filament elongation. However, as no metabolic alteration in JA-Ile turnover was evidenced in total flower extracts of CYP94B1-deficient plants, identifying the exact substrate of CYP94B1 will require analysis in isolated organs. The specific expression of CYP94C1 in dehisced anthers is consistent with a termination of JA-Ile signaling that is active in this organ. From genetic data we infer that most 12COOH-JA-Ile measured in stage 14 flowers originates from JA-Ile and $12 \mathrm{OH}-\mathrm{JA}-\mathrm{Ile}$ oxidation in anthers. These expression patterns suggest a sophisticated organization of JA-Ile control, where an increasing oxidation gradient from the base of filament toward the anther may be coupled with the transport of intermediate forms. Consistent with unchanged JA-Ile levels, CYP-deficient lines did not display altered expression of target genes, including JAZ, MYB21/24 or the defensive myrosinases TGG (data not shown). The molecular function of active JA-Ile catabolism at late stamen development is thus not clear. A future challenge will be 
to map the sites of accumulation and conversion of JA/JA-Ile metabolites in Arabidopsis flowers to understand JA dynamics in reproductive development.

\subsection{Comparative Analysis of JA Pools in Flower Development and Leaf Stress Responses}

JA signaling plays important roles in many aspects of plant life. We established that the master regulator JA-Ile is at the crossroads of a complex metabolic grid whose dynamics are highly controlled to fine-tune flexible stress or developmental responses (Figure 1). In recent years we developed genetic and analytical tools to investigate the metabolic fate of JA-Ile in three distinct models, flower development, as described above, and two types of leaf stress responses reported previously: mechanical wounding $[16,21,24]$ and infection by the necrotrophic fungus Botrytis cinerea [15], which reflect processes activated upon plant-insect and plant-microbe interactions, respectively.

In flowers, endogenous signals involving sequential hormonal cascades upregulate JA synthesis which then activates coordinated developmental processes that end up in the timely release of fertile pollen [50]. Upon leaf biotic stress, defense responses can be induced by two distinct and antagonistic branches of the JA/JA-Ile pathway [51]. On one hand, the attack by herbivorous insects, which can be partially mimicked by mechanical wounding, provokes a massive synthesis of JAs [52] where JA-Ile signals synergistically with abscisic acid (ABA) and de-represses MYC2-dependent transcription of defense genes [53,54]. On the other hand, resistance to necrotrophic microbes relies on the concomitant activation of JA and ethylene signals that are integrated by the AP2/ERF/ORA59 transcription factors. In these three biological contexts, the JA metabolic pathway receives input from different upstream activation factors, which translates into particular JA profiles/dynamics, and ultimately regulates distinct sets of target genes.

In the next section, we comment on quantitative and qualitative differences in JA homeostasis in these different models. We focused on the temporal distribution of a set of 5 relevant JAs across these biological systems, although the complement of JA derivatives undoubtedly extends to many more compounds. A synthetic view of the compared data is represented in Figure 4 in the form of pie diagrams. In the first column the cumulated amount of these compounds (JA, 12OH-JA, JA-Ile, 12OH-JA-Ile and 12COOH-JA-Ile) is given along with their relative distribution (in percentage). The middle and right column depict respectively the amino acid-conjugation and $\omega$-oxidation status of the JA blend monitored in each analysis.

\subsubsection{JA Profile Evolution upon Flower Opening}

Among the material we examined, the flower samples displayed the lowest content in JAs, with a total around $3 \mathrm{nmol} \cdot \mathrm{g} \cdot \mathrm{FW}^{-1}$. This result has likely two origins: the collection method combined in one sample all stages of closed flower buds (stages 1-12), including early stages where JA synthesis may not have started. In addition, extracting JAs from total (undissected) buds as performed in this study did not give access to organ-specific signal that was likely diluted by neighboring tissues that are poor in JAs. As a net result, JA, $12 \mathrm{OH}-\mathrm{JA}$ and $12 \mathrm{COOH}-\mathrm{JA}$-Ile were found equally abundant in closed buds, followed by JA-Ile, illustrating that major steps in JA metabolism are operating before anthesis (Figure 4A). Upon floral opening, two major changes are detected in WT flowers: a 5-fold reduction in JA-Ile and an approximately 4 -fold increase of $12 \mathrm{COOH}-\mathrm{JA}-\mathrm{Ile}$. As a consequence, in open flowers, there is a tremendous shift from JA-Ile towards a large pool of $12 \mathrm{COOH}-J A-I l e$, the ratio between these two compounds reaching about 1 to 75 (Figure 4). 


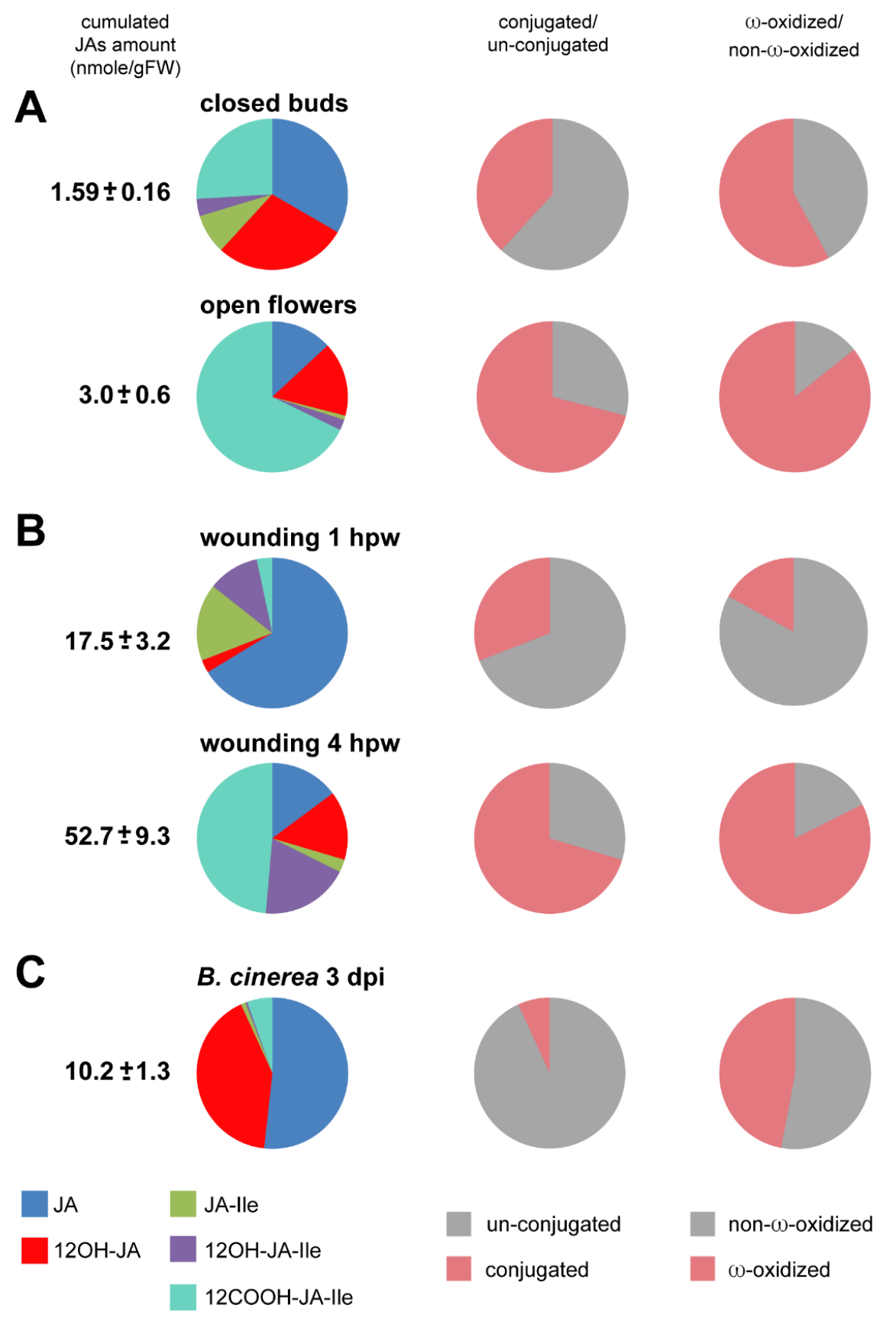

Figure 4. Pie diagrams of five JAs content in three biological systems: In left column, the number indicates cumulated amounts (nmol. $\mathrm{g} \cdot \mathrm{FW}^{-1}$ ) of JA, 12OH-JA, JA-Ile, 12OH-JA-Ile and $12 \mathrm{COOH}-J A-I l e$ as means with SD of three independent biological repeats. The left column pies indicate the proportions of conjugated (JA-Ile + 12OH-JA-Ile + 12COOH-JA-Ile) versus un-conjugated $(\mathrm{JA}+12 \mathrm{OH}-\mathrm{JA})$. The right column pies indicate the proportions of non- $\omega$-oxidized (JA + JA-Ile) versus $\omega$-oxidized (12OH-JA + 12OH-JA-Ile + 12COOH-JA-Ile) JAs. (A) flower maturation: closed buds or open flowers (B) leaf wounding: $1 \mathrm{~h}$ post-wounding (hpw) or $4 \mathrm{hpw}(\mathbf{C})$ leaf B. cinerea infection: 3 days post-inoculation (dpi).

\subsubsection{JA Profile Evolution upon Mechanical Wounding}

Mechanical wounding is a convenient laboratory model to trigger massive transcriptional responses reminiscent of those activated by an herbivore insect attack. It provides a severe and 
synchronous stimulus that rapidly activates JA synthesis, signaling, and catabolism [9]. Hence, the cumulated amounts of the 6 analyzed JAs reach about $50 \mathrm{nmol} \cdot \mathrm{g} \cdot \mathrm{FW}^{-1}$ at $4 \mathrm{~h}$ post-wounding (hpw).

In this material, stimulated cells undergo waves of molecular events that can be distinguished over time. Here we used results previously published in [16] and [24] and extracted data at the $1^{\text {st }}$ and $4^{\text {th }} \mathrm{hpw}$ that correspond to two different stages of the JA metabolic response (Figure 4B). At $1 \mathrm{hpw}$ JA-Ile is at its peak level [16], JA is the most abundant of the 5 compounds while 12OH-JA and 12OH-JA-Ile are still minor oxidized JAs. Overall, at this early time point, JAs are mainly unconjugated and unoxidized. At $4 \mathrm{hpw}$, a strong shift toward $\omega$-oxidized and conjugated derivatives accumulation has occurred, 12COOH-JA-Ile representing nearly half of all compounds. This is reflective of the coordinated activation of conjugation/deconjugation and $\omega$-oxidation pathways $[16,24]$ that channel main fluxes toward the accumulation of downstream JAs while depleting the active hormone JA-Ile. The $4 \mathrm{hpw}$ blend resembles qualitatively the distribution in open flowers, and is characteristic of the JA-Ile signal extinction phase.

\subsubsection{JA Profile in B. cinerea-Infected Leaves}

Here the data are based on our previous investigation of JA profiles in response to Botrytis infection and the impact of CYP94 expression as described in [15]. Upon fungal infection, the dynamics of leaf stimulation are different from wounding as disease lesions develop radially starting from a single inoculation site on each half leaf. In this case, new tissues are stimulated continuously, therefore asynchronously, making this system less suitable to dissect the dynamics of metabolic conversions, sampled leaves combining both early and late metabolic events. Consequently, JA accumulation merely evolves quantitatively with lesion size, with little change in species distribution over time. A snapshot at $3 \mathrm{dpi}$ of the JA profile in fungus-infected leaves is given in Figure 4C. The total 5-JA pool culminates here at about $10 \mathrm{nmol} \cdot \mathrm{g} \cdot \mathrm{FW}^{-1}$ in leaves bearing two infection sites. Here, JA and 12OH-JA represent together $>90 \%$ of all analyzed JAs. Oxidation is less prevalent compared to flowers or wounded leaves. Another striking feature of this profile is that unconjugated JAs dominate upon B. cinerea infection, and JA-amino acid conjugates and their derivatives remain minor in proportion. A possibility is that conjugating activity is low, or it may be reversed by high amido-hydrolase activity, for example IAR3 and ILL6 that generate JA and 12OH-JA [24]. Consequently, a large part of JA remains unmodified in reaction to $B$. cinerea infection.

\section{Experimental Section}

\subsection{Plant Material and Growth Conditions}

All Arabidopsis genotypes used in this paper were in Col0 ecotype and were grown under a $12 \mathrm{~h}$ light $/ 12 \mathrm{~h}$ dark photoperiod in a growth chamber $\left(21 / 18^{\circ} \mathrm{C}\right.$, respectively). T-DNA insertion lines were obtained from NASC center: cyp94b1-1 (SALK_129672), cyp94b3-1 (CS302217), cyp94c1-1 (SALK_55455). Double and triple mutants were obtained by crossing of corresponding original homozygous lines with subsequent analysis of T2 generation [15]. CYP94C1 overexpressing line (C1-OE) was described in [16].

\subsection{Jasmonate Profiles}

Extraction of jasmonates and their quantification by UPLC-MS/MS were performed as described in [24].

\subsection{RNA Extraction and Real-Time PCR}

RNA extraction, reverse transcription and Reverse Transcriptase-qPCR analysis were performed as described in [16] using about $80 \mathrm{mg}$ of whole flower material and $30 \mathrm{mg}$ for samples of dissected flowers. 


\subsection{Histochemical GUS Staining}

pCYP94C1::GUS lines were provided by Yves Millet (Massachussets General Hospital, Boston, MA, USA). $p$ CYP94B1::GUS and $p$ CYP94D2::GUS constructs were obtained by cloning of promoter sequences ranging from -1587 to $-55 \mathrm{bp}$ and from -1871 to $-15 \mathrm{bp}$ of corresponding genes, respectively (the A in ATG start codon is refered as +1 ), in pBGWFS7 vector with subsequent transformation of Col0 plants. All material used for staining was from homozygous plants. GUS coloration was performed as described in [55] and samples were observed with a Leica Z16 APOA macroscope.

\subsection{Statistical Analysis}

Statistical analysis was performed using Infostat software (http://www.infostat.com.ar, Universidad Nacional de Córdoba, Argentina). Comparisons of sample medians were performed by Kruskal-Wallis one-way analysis of variance (ANOVA) $(p<0.05)$ to determine whether sample means were significantly different.

\section{Conclusions}

The analysis of JA-Ile turnover in Arabidopsis flowers further extends the involvement of CYP94 and amidohydrolase enzymes in the control of hormone turnover to a developmental case of JA signaling. Hormone analysis revealed highly active JA-Ile catabolism in maturing stamens, in accordance with extensive genetic data underlying the importance of jasmonates in male fertility in Arabidopsis. CYP94C1 was identified as the predominant enzyme for JA-Ile oxidative turnover, but surprisingly, the three studied CYP94 JA-Ile oxidases are dispensable for the rapid removal of JA-Ile upon flower anthesis, despite the large metabolic flux proceeding through JA-Ile oxidation. These observations open the possibility of alternative JA-Ile clearance route(s) in flowers. Based on the characterization of some enzymatic actors and likely sites of JA-Ile turnover, one of the next challenges will be to visualize specific JAs in flower organs and to elucidate how JA metabolism affects hormonal functions in plant reproduction.

The comparison of the dynamics of JA metabolism in flowers, a developmental model, with two previously characterized models of induced leaf defense, illustrates the great metabolic plasticity in the JA pathway. Even though JA-Ile is a central signal in all three systems, and common catabolic pathways are activated in the three situations, pools of individual JAs are very different, reflecting distinct bottlenecks in each physiological context. These specific features are highlighted in the pathway depicted in Figure 1, with variable arrow thicknesses reflecting presumed flux through each reaction. Additional parameters, such as interactions with other hormones and yet unknown routes in JA-Ile homeostasis may be crucial. Their search, together with new regulatory functions associated with JA metabolites will be the topic of new exciting investigations.

Acknowledgments: We thank Franck Pinot for valuable discussions. This work was funded by a doctoral fellowship from the Ministère de l'Enseignement Supérieur et de la Recherche to EW and partially by the Agence Nationale de la Recherche (contract ANR-12-BSV8-005 for Y.A. and E.S.).

Author Contributions: E. W. performed flower metabolic and gene expression analysis; E. S. drafted the manuscript and assembled figures; Y. A. generated pCYP94B1::GUS and pCYP94D2::GUS constructs and transformed plants. L. M. synthesized the standard compounds for jasmonate determinations. T. H. supervised the work and finalized the manuscript.

Conflicts of Interest: The authors declare no conflict of interest.

\section{References}

1. Acosta, I.F.; Farmer, E.E. Jasmonates. Arabidopsis Book 2010, 8, e0129. [CrossRef] [PubMed]

2. Fonseca, S.; Chico, J.M.; Solano, R. The jasmonate pathway: The ligand, the receptor and the core signalling module. Curr. Opin. Plant. Biol. 2009, 12, 539-547. [CrossRef] [PubMed] 
3. Wasternack, C.; Kombrink, E. Jasmonates: Structural requirements for lipid-derived signals active in plant stress responses and development. ACS Chem. Biol. 2010, 5, 63-77. [CrossRef] [PubMed]

4. Wasternack, C.; Hause, B. Jasmonates: Biosynthesis, perception, signal transduction and action in plant stress response, growth and development. An update to the 2007 review in Annals of Botany. Ann. Bot. 2013, 111, 1021-1058. [CrossRef] [PubMed]

5. Schaller, A.; Stintzi, A. Enzymes in jasmonate biosynthesis-structure, function, regulation. Phytochemistry 2009, 70, 1532-1538. [CrossRef] [PubMed]

6. Ellinger, D.; Stingl, N.; Kubigsteltig, I.I.; Bals, T.; Juenger, M.; Pollmann, S.; Berger, S.; Schuenemann, D.; Mueller, M.J. DONGLE and DEFECTIVE IN ANTHER DEHISCENCE1 lipases are not essential for woundand pathogen-induced jasmonate biosynthesis: Redundant lipases contribute to jasmonate formation. Plant Physiol. 2010, 153, 114-127. [CrossRef] [PubMed]

7. Ishiguro, S.; Kawai-Oda, A.; Ueda, J.; Nishida, I.; Okada, K. The DEFECTIVE IN ANTHER DEHISCIENCE gene encodes a novel phospholipase A1 catalyzing the initial step of jasmonic acid biosynthesis, which synchronizes pollen maturation, anther dehiscence, and flower opening in Arabidopsis. Plant Cell 2001, 13, 2191-2209. [CrossRef] [PubMed]

8. Koo, A.J.; Howe, G.A. Catabolism and deactivation of the lipid-derived hormone jasmonoyl-isoleucine. Front. Plant Sci. 2012, 3, 19. [CrossRef] [PubMed]

9. Heitz, T.; Smirnova, E.; Widemann, E.; Aubert, Y.; Pinot, F.; Ménard, R. The rise and fall of jasmonate biological activities. In Lipids in Plant and Algae Development; Nakamura, Y., Li-Beisson, Y., Eds.; Springer: Berlin, Germany, 2016; Volume 86.

10. Fonseca, S.; Chini, A.; Hamberg, M.; Adie, B.; Porzel, A.; Kramell, R.; Miersch, O.; Wasternack, C.; Solano, R. (+)-7-Iso-Jasmonoyl-L-isoleucine is the endogenous bioactive jasmonate. Nat. Chem. Biol. 2009, 5, 344-350. [CrossRef] [PubMed]

11. Thines, B.; Katsir, L.; Melotto, M.; Niu, Y.; Mandaokar, A.; Liu, G.; Nomura, K.; He, S.Y.; Howe, G.A.; Browse, J. JAZ repressor proteins are targets of the $\mathrm{SCF}(\mathrm{COI} 1)$ complex during jasmonate signalling. Nature 2007, 448, 661-665. [CrossRef] [PubMed]

12. Gimenez-Ibanez, S.; Boter, M.; Solano, R. Novel players fine-tune plant trade-offs. Essays Biochem. 2015, 58, 83-100. [CrossRef] [PubMed]

13. Miersch, O.; Neumerkel, J.; Dippe, M.; Stenzel, I.; Wasternack, C. Hydroxylated jasmonates are commonly occurring metabolites of jasmonic acid and contribute to a partial switch-off in jasmonate signaling. New Phytol. 2008, 177, 114-127. [CrossRef] [PubMed]

14. Huot, B.; Yao, J.; Montgomery, B.L.; He, S.Y. Growth-defense tradeoffs in plants: a balancing act to optimize fitness. Mol. Plant 2014, 7, 1267-1287. [CrossRef] [PubMed]

15. Aubert, Y.; Widemann, E.; Miesch, L.; Pinot, F.; Heitz, T. CYP94-mediated jasmonoyl-isoleucine hormone oxidation shapes jasmonate profiles and attenuates defence responses to Botrytis cinerea infection. J. Exp. Bot. 2015, 66, 3879-3892. [CrossRef] [PubMed]

16. Heitz, T.; Widemann, E.; Lugan, R.; Miesch, L.; Ullmann, P.; Desaubry, L.; Holder, E.; Grausem, B.; Kandel, S.; Miesch, M.; et al. Cytochromes P450 CYP94C1 and CYP94B3 catalyze two successive oxidation steps of plant hormone Jasmonoyl-isoleucine for catabolic turnover. J. Biol. Chem. 2012, 287, 6296-6306. [CrossRef] [PubMed]

17. Kitaoka, N.; Matsubara, T.; Sato, M.; Takahashi, K.; Wakuta, S.; Kawaide, H.; Matsui, H.; Nabeta, K.; Matsuura, H. Arabidopsis CYP94B3 encodes jasmonyl-L-isoleucine 12-hydroxylase, a key enzyme in the oxidative catabolism of jasmonate. Plant Cell Physiol. 2011, 52, 1757-1765. [CrossRef] [PubMed]

18. Koo, A.J.; Cooke, T.F.; Howe, G.A. Cytochrome P450 CYP94B3 mediates catabolism and inactivation of the plant hormone jasmonoyl-L-isoleucine. Proc. Natl. Acad. Sci. USA 2011, 108, 9298-9303. [CrossRef] [PubMed]

19. Koo, A.J.; Thireault, C.; Zemelis, S.; Poudel, A.N.; Zhang, T.; Kitaoka, N.; Brandizzi, F.; Matsuura, H.; Howe, G.A. Endoplasmic reticulum-associated inactivation of the hormone jasmonoyl-L-isoleucine by multiple members of the cytochrome P450 94 family in Arabidopsis. J. Biol. Chem. 2014, 289, 29728-29738. [CrossRef] [PubMed] 
20. Kitaoka, N.; Kawaide, H.; Amano, N.; Matsubara, T.; Nabeta, K.; Takahashi, K.; Matsuura, H. CYP94B3 activity against jasmonic acid amino acid conjugates and the elucidation of 12-O-beta-glucopyranosyljasmonoyl-L-isoleucine as an additional metabolite. Phytochemistry 2014, 99, 6-13. [CrossRef] [PubMed]

21. Widemann, E.; Grausem, B.; Renault, H.; Pineau, E.; Heinrich, C.; Lugan, R.; Ullmann, P.; Miesch, L.; Aubert, Y.; Miesch, M.; et al. Sequential oxidation of Jasmonoyl-Phenylalanine and Jasmonoyl-Isoleucine by multiple cytochrome P450 of the CYP94 family through newly identified aldehyde intermediates. Phytochemistry 2015, 117, 388-399. [CrossRef] [PubMed]

22. Woldemariam, M.G.; Onkokesung, N.; Baldwin, I.T.; Galis, I. Jasmonoyl-L-isoleucine hydrolase 1 (JIH1) regulates jasmonoyl-L-isoleucine levels and attenuates plant defenses against herbivores. Plant J. 2012, 72, 758-767. [CrossRef] [PubMed]

23. Rampey, R.A.; LeClere, S.; Kowalczyk, M.; Ljung, K.; Sandberg, G.; Bartel, B. A family of auxin-conjugate hydrolases that contributes to free indole-3-acetic acid levels during Arabidopsis germination. Plant Physiol. 2004, 135, 978-988. [CrossRef] [PubMed]

24. Widemann, E.; Miesch, L.; Lugan, R.; Holder, E.; Heinrich, C.; Aubert, Y.; Miesch, M.; Pinot, F.; Heitz, T. The amidohydrolases IAR3 and ILL6 contribute to jasmonoyl-isoleucine hormone turnover and generate 12-hydroxyjasmonic acid upon wounding in Arabidopsis leaves. J. Biol. Chem. 2013, 288, 31701-31714. [CrossRef] [PubMed]

25. McConn, M.; Browse, J. The Critical Requirement for Linolenic Acid Is Pollen Development, Not Photosynthesis, in an Arabidopsis Mutant. Plant Cell 1996, 8, 403-416. [CrossRef] [PubMed]

26. Park, J.H.; Halitschke, R.; Kim, H.B.; Baldwin, I.T.; Feldmann, K.A.; Feyereisen, R. A knock-out mutation in allene oxide synthase results in male sterility and defective wound signal transduction in Arabidopsis due to a block in jasmonic acid biosynthesis. Plant J. 2002, 31, 1-12. [CrossRef] [PubMed]

27. Sanders, P.M.; Lee, P.Y.; Biesgen, C.; Boone, J.D.; Beals, T.P.; Weiler, E.W.; Goldberg, R.B. The Arabidopsis DELAYED DEHISCENCE1 gene encodes an enzyme in the jasmonic acid synthesis pathway. Plant Cell 2000, 12, 1041-1061. [CrossRef] [PubMed]

28. Schilmiller, A.L.; Koo, A.J.; Howe, G.A. Functional diversification of acyl-coenzyme A oxidases in jasmonic acid biosynthesis and action. Plant Physiol. 2007, 143, 812-824. [CrossRef] [PubMed]

29. Stintzi, A.; Browse, J. The Arabidopsis male-sterile mutant, opr3, lacks the 12-oxophytodienoic acid reductase required for jasmonate synthesis. Proc. Natl. Acad. Sci. USA 2000, 97, 10625-10630. [CrossRef] [PubMed]

30. Xie, D.X.; Feys, B.F.; James, S.; Nieto-Rostro, M.; Turner, J.G. COI1: An Arabidopsis gene required for jasmonate-regulated defense and fertility. Science 1998, 280, 1091-1094. [CrossRef] [PubMed]

31. Nelson, M.R.; Band, L.R.; Dyson, R.J.; Lessinnes, T.; Wells, D.M.; Yang, C.; Everitt, N.M.; Jensen, O.E.; Wilson, Z.A. A biomechanical model of anther opening reveals the roles of dehydration and secondary thickening. New Phytol. 2012, 196, 1030-1037. [CrossRef] [PubMed]

32. Smyth, D.R.; Bowman, J.L.; Meyerowitz, E.M. Early flower development in Arabidopsis. Plant Cell 1990, 2, 755-767. [CrossRef] [PubMed]

33. Reeves, P.H.; Ellis, C.M.; Ploense, S.E.; Wu, M.F.; Yadav, V.; Tholl, D.; Chetelat, A.; Haupt, I.; Kennerley, B.J.; Hodgens, C.; et al. A regulatory network for coordinated flower maturation. PLoS Genet. 2012, 8, e1002506. [CrossRef] [PubMed]

34. Yuan, Z.; Zhang, D. Roles of jasmonate signalling in plant inflorescence and flower development. Curr. Opin. Plant Biol. 2015, 27, 44-51. [CrossRef] [PubMed]

35. Qi, T.; Huang, H.; Song, S.; Xie, D. Regulation of jasmonate-mediated stamen development and seed production by a bHLH-MYB complex in Arabidopsis. Plant Cell 2015, 27, 1620-1633. [CrossRef] [PubMed]

36. Song, S.; Qi, T.; Huang, H.; Ren, Q.; Wu, D.; Chang, C.; Peng, W.; Liu, Y.; Peng, J.; Xie, D. The Jasmonate-ZIM domain proteins interact with the R2R3-MYB transcription factors MYB21 and MYB24 to affect Jasmonate-regulated stamen development in Arabidopsis. Plant Cell 2011, 23, 1000-1013. [CrossRef] [PubMed]

37. Mandaokar, A.; Thines, B.; Shin, B.; Lange, B.M.; Choi, G.; Koo, Y.J.; Yoo, Y.J.; Choi, Y.D.; Choi, G.; Browse, J. Transcriptional regulators of stamen development in Arabidopsis identified by transcriptional profiling. Plant J. 2006, 46, 984-1008. [CrossRef] [PubMed]

38. Cai, Q.; Yuan, Z.; Chen, M.; Yin, C.; Luo, Z.; Zhao, X.; Liang, W.; Hu, J.; Zhang, D. Jasmonic acid regulates spikelet development in rice. Nat. Commun. 2014, 5, 3476. [CrossRef] [PubMed] 
39. Lee, S.H.; Sakuraba, Y.; Lee, T.; Kim, K.W.; An, G.; Lee, H.Y.; Paek, N.C. Mutation of Oryza sativa CORONATINE INSENSITIVE $1 \mathrm{~b}$ (OsCOI1b) delays leaf senescence. J. Integr. Plant Biol. 2015, 57, 562-576. [CrossRef] [PubMed]

40. Xiao, Y.; Chen, Y.; Charnikhova, T.; Mulder, P.P.; Heijmans, J.; Hoogenboom, A.; Agalou, A.; Michel, C.; Morel, J.B.; Dreni, L.; et al. OsJAR1 is required for JA-regulated floret opening and anther dehiscence in rice. Plant Mol. Biol. 2014, 86, 19-33. [CrossRef] [PubMed]

41. Acosta, I.F.; Laparra, H.; Romero, S.P.; Schmelz, E.; Hamberg, M.; Mottinger, J.P.; Moreno, M.A.; Dellaporta, S.L. Tasselseed1 is a lipoxygenase affecting jasmonic acid signaling in sex determination of maize. Science 2009, 323, 262-265. [CrossRef] [PubMed]

42. Yan, Y.; Christensen, S.; Isakeit, T.; Engelberth, J.; Meeley, R.; Hayward, A.; Emery, R.J.; Kolomiets, M.V. Disruption of OPR7 and OPR8 reveals the versatile functions of jasmonic acid in maize development and defense. Plant Cell 2012, 24, 1420-1436. [CrossRef] [PubMed]

43. Li, L.; Zhao, Y.; McCaig, B.C.; Wingerd, B.A.; Wang, J.; Whalon, M.E.; Pichersky, E.; Howe, G.A. The tomato homolog of CORONATINE-INSENSITIVE1 is required for the maternal control of seed maturation, jasmonate-signaled defense responses, and glandular trichome development. Plant Cell 2004, 16, 126-143. [CrossRef] [PubMed]

44. Goetz, S.; Hellwege, A.; Stenzel, I.; Kutter, C.; Hauptmann, V.; Forner, S.; McCaig, B.; Hause, G.; Miersch, O.; Wasternack, C.; Hause, B. Role of cis-12-oxo-phytodienoic acid in tomato embryo development. Plant Physiol. 2012, 158, 1715-1727. [CrossRef] [PubMed]

45. Stitz, M.; Hartl, M.; Baldwin, I.T.; Gaquerel, E. Jasmonoyl-1-isoleucine coordinates metabolic networks required for anthesis and floral attractant emission in wild tobacco (Nicotiana attenuata). Plant Cell 2014, 26, 3964-3983. [CrossRef] [PubMed]

46. Wasternack, C.; Forner, S.; Strnad, M.; Hause, B. Jasmonates in flower and seed development. Biochimie 2013, 95, 79-85. [CrossRef] [PubMed]

47. Hause, B.; Stenzel, I.; Miersch, O.; Maucher, H.; Kramell, R.; Ziegler, J.; Wasternack, C. Tissue-specific oxylipin signature of tomato flowers: Allene oxide cyclase is highly expressed in distinct flower organs and vascular bundles. Plant J. 2000, 24, 113-126. [CrossRef] [PubMed]

48. Liu, Z.; Boachon, B.; Lugan, R.; Tavares, R.; Erhardt, M.; Mutterer, J.; Demais, V.; Pateyron, S.; Brunaud, V.; Ohnishi, T.; et al. A conserved cytochrome P450 evolved in seed plants regulates flower maturation. Mol. Plant 2015, 8, 1751-1765. [CrossRef] [PubMed]

49. Stenzel, I.; Otto, M.; Delker, C.; Kirmse, N.; Schmidt, D.; Miersch, O.; Hause, B.; Wasternack, C. ALLENE OXIDE CYCLASE (AOC) gene family members of Arabidopsis thaliana: Tissue- and organ-specific promoter activities and in vivo heteromerization. J. Exp. Bot. 2012, 63, 6125-6138. [CrossRef] [PubMed]

50. Song, S.; Qi, T.; Huang, H.; Xie, D. Regulation of stamen development by coordinated actions of jasmonate, auxin, and gibberellin in Arabidopsis. Mol. Plant 2013, 6, 1065-1073. [CrossRef] [PubMed]

51. Verhage, A.; Vlaardingerbroek, I.; Raaymakers, C.; Van Dam, N.M.; Dicke, M.; Van Wees, S.C.; Pieterse, C.M. Rewiring of the Jasmonate Signaling Pathway in Arabidopsis during Insect Herbivory. Front. Plant Sci. 2011, 2, 47. [CrossRef] [PubMed]

52. Glauser, G.; Grata, E.; Dubugnon, L.; Rudaz, S.; Farmer, E.E.; Wolfender, J.L. Spatial and temporal dynamics of jasmonate synthesis and accumulation in Arabidopsis in response to wounding. J. Biol. Chem. 2008, 283, 16400-16407. [CrossRef] [PubMed]

53. Kazan, K.; Manners, J.M. Jasmonate signaling: toward an integrated view. Plant Physiol. 2008, 146, 1459-1468. [CrossRef] [PubMed]

54. Lorenzo, O.; Chico, J.M.; Sanchez-Serrano, J.J.; Solano, R. JASMONATE-INSENSITIVE1 encodes a MYC transcription factor essential to discriminate between different jasmonate-regulated defense responses in Arabidopsis. Plant Cell 2004, 16, 1938-1950. [CrossRef] [PubMed]

55. La Camera, S.; Geoffroy, P.; Samaha, H.; Ndiaye, A.; Rahim, G.; Legrand, M.; Heitz, T. A pathogen-inducible patatin-like lipid acyl hydrolase facilitates fungal and bacterial host colonization in Arabidopsis. Plant J. 2005, 44, 810-825. [CrossRef] [PubMed]

(C) 2016 by the authors; licensee MDPI, Basel, Switzerland. This article is an open access article distributed under the terms and conditions of the Creative Commons by Attribution (CC-BY) license (http://creativecommons.org/licenses/by/4.0/). 